\title{
SEMI SEPARATION AXIOMS AND HYPERSPACES
}

\author{
CHARLES DORSETT \\ Department of Mathematics, Texas A\&M University \\ College Station, Texas
}

(Received April 21, 1980 and in revised form September 4, 1980)

ABSTRACT. In this paper examples are given to show that s-regular and s-normal are independent; that s-normal, and s-regular are not semi topological properties; and that $(S(X), E(X))$ need not be semi-T 1 even if $(X, T)$ is compact, s-normal, s-regular, semi-T $T_{2}$, and $T_{0}$. Also, it is shown that for each space $(X, T)$, $(\mathrm{S}(\mathrm{X}), \mathrm{E}(\mathrm{X})),\left(\mathrm{S}\left(\mathrm{X}_{0}\right), \mathrm{E}\left(\mathrm{X}_{0}\right)\right)$, and $\left(\mathrm{S}\left(\mathrm{X}_{\mathrm{SO}}\right), \mathrm{E}\left(\mathrm{X}_{\mathrm{S} 0}\right)\right)$ are homeomorphic, where $\left(X_{0}, Q\left(X_{0}\right)\right)$ is the $T_{0}$-identification space of $(X, T)$ and $\left(X_{S 0}, Q\left(X_{S 0}\right)\right)$ is the semi-T $T_{0}$-identification space of $(X, T)$, and that if $(X, T)$ is $s$-regular and $R_{0}$, then $(S(X), E(X))$ is semi-T ${ }_{2}$.

KEY WORDS AND PHRASES. Semi open sets, semi topological properties, and hyperspaces.

1980 MATHEMATICS SUBJECT CLASSIFICATION CODES. 54A10, 54820.

1. INTRODUCTION.

Semi open sets were first defined and investigated by Levine [1] in 1963.

DEFINITION 1.1. Let $(X, T)$ be a space and let $A \in X$. Then $A$ is semi open, denoted by $A \in S O(X, T)$, 1ff there exists $U \in T$ such that $U \subset A \subset \bar{U}$.

Since 1963 semi open sets have been used to define and investigate many new topological properties. Maheshwari and Prasad [2], [3], and [4] generalized $T_{1}$, $i=0,1,2$, regular, and normal to semi-T $1,1=0,1,2, s$-regular, and s-normal, 
by replacing the word open in the definitions of $T_{1}, 1=0,1,2$, regular, and normal by semi open, respectively. Except for s-normal and s-regular, the relationships between these separation axioms have been determined. In this paper, the relationship between s-normal and s-regular is determined, and semi topological properties and hyperspaces are further investigated.

\section{S-REGULAR, \&-NORMAL, AND SEMI TOPOLOGICAL PROPERTIES.}

Maheshwari and Prasad [4] gave an example showing that s-normal does not imply s-regular. That example can be combined with the following example to show that s-regular and s-normal are independent.

EXAMPLE 2.1 Let $\mathrm{N}$ denote the natural numbers, let $\mathrm{T}$ be the discrete topology on $N$, let e be the embedding map of $(N, T)$ into $\pi\left\{I_{f} \mid f \in C *(N, T)\right\}$, and let $(\beta N, W)=(\overline{e(N)}, e)$ denote the Stone-Čech compactification of $(N, T)$. From Willard's book [5], ( $B N, W)$ is extremely disconnected, $e(N)$ is open in $B N$, and $B=\beta N-e(N)$ is infinite. For each $p \in N$ let $N_{p}=\{n \in N \mid n \leq p\}$. Since for each $p \in N$, there exists a function $f_{p}: N_{p} \rightarrow B \times W$ such that (1) if $f \in\{2, \ldots, p\}$, then $f_{i}$ is an extension of $f_{1-1}$, (2) $x_{1} \in 0_{i}$ for all $i \in N_{p}$, (3) if 1 , $j \in N_{p}$, then $\overline{0}_{1} n^{\prime} \overline{0}_{j} \neq \emptyset$ iff $1=j$, and (4) B- $\stackrel{P}{i=1}_{1} \overline{0}_{1}$ is infinite, then there exists a sequence $\left\{\left(x_{n}, 0_{n}\right)\right\}_{n \in N} \subset B \times W$ such that $x_{n} \in 0_{n}$ for all $n \in N$ and $\overline{0}_{m} \gamma_{1} \overline{0}_{n} \neq \emptyset$ iff $m=n$. Let $\left\{a_{n}\right\}_{n \in N}$ be a sequence such that $\left\{a_{n} \mid n \in N\right\} \Gamma_{1 \beta N}=\emptyset$ and $a_{n}=a_{m}$ iff $n=m$, let $v=\left\{x_{n} \mid n \in N\right\} \cup\left({ }_{n} U_{N}\left\{U_{n}=0_{n} \cap e(N)\right\}\right) c_{\beta N}$, let $W_{1}$ be the relative topology on $V$, and let $x=V U\left\{a_{n} \mid n \in N\right\}$. Since $U_{1}$ is countably infinite, then $U_{1}=\left\{y_{n}\right\}_{n \in N}$, where $y_{i}=y_{j}$ iff $i=j$. For each $i \in N$, let $B_{i}=\left\{0 \subset x-\left(\left\{x_{n} \mid n \in N\right\} \cup\left\{a_{n} \mid n \neq i\right\}\right) \mid 0 \Gamma_{i} U_{n} \in w_{1}\right.$ for all $n \in N, x_{n} \in \overline{0 \sqrt{U_{n}}}$ except for finitely many $n \in N$, and $\left.a_{i}, y_{i} \in 0\right\}$, and let $W_{2}=\underset{i \in N}{U} B_{i}$. Then $W_{1} \cup W_{2}$ is a base for a topology $S$ on $X,(X, S)$ is s-regular, semi-T $T_{2}$, and $T_{0}$, and $(X, S)$ is not s-normal since $A=\left\{a_{n} \mid n \in N\right\}$ and $C=\left\{x_{n} \mid n \in N\right\}$ are disjoint closed sets and there do not exist disjoint semi open sets containing $A$ and $C$, respectively.

Semihomeomorphisms and semi topological properties were first introduced and investigated by Crossley and Hildebrand [6]. 
DEFINITION 2.1. A 1-1 function from one space onto another space is a semihomeomorphism iff images of semi open sets are semi open and inverses of semi open sets are semi open. A property of topological spaces preserved by semihomeomorphisms is called a semi topological property.

Example 1.5 in [6], which was used to show that normal and regular are not semi topological properties, also shows that s-normal and s-regular are not semi topological properties.

Clearly, semi-T $i, i=0,1,2$, are semi topological properties.

\section{HYPERSPACES AND SEMI SEPARATION AXIOMS}

DEFINITION 3.1. Let $(X, T)$ be a topological space, let $A \subset X$, and define $S(X), S(A)$, and $I(A)$ as follows: $S(X)=\{F \subset X \mid F$ is nonempty and closed $\}$, $S(A)=\{F \in S(X) \mid F \subset A\}$, and $I(A)=\{F \in S(X) \mid F\lceil i A \neq \emptyset\}$. Denote by $E(X)$ the smallest topology on $S(X)$ satisfying the conditions that if $G \in T$, then $S(G) \in E(X)$ and $I(G) \in E(X)$. Then $(S(X), E(X))$ is called a hyperspace [7]. Michael [8] showed that for a space $(X, T), B=\left\{\left\langle G_{1}, \ldots, G_{p}\right\rangle \mid p \in N\right.$ and $G_{i} \in T$ for all $\left.1 \in N_{p}=\{1, \downarrow \ldots, p\}\right\}$ is a base for $E(X)$, where $N$ is the natural numbers and $\left\langle G_{1}, \ldots, G_{p}\right\rangle=\left\langle G_{i}\right\rangle_{i=1}^{p}=\left\{F \in S(X) \mid F \subset \bigcup_{i=1}^{P} G_{1}\right.$ and $F \cap G_{i} \neq \emptyset$ for all $\left.i \in N_{p}\right\}$, and observed that for each space $(X, Y)$, $(S(X), E(X))$ is $T_{0}$. Since $T_{0}$ implies semi-T , then for each space $(X, T)$, $(S(X), E(X))$ is semi- $T_{0}$. The following example shows that $(S(X), E(X))$ need not be semi-T $T_{1}$ even if $(X, T)$ is compact, s-normal, s-regualr, semi- $T_{2}$, and $T_{0}$.

EXAMPLE 3.1 Let $X=\{a, b, c, d\}$ and $T=\{X, \phi,\{b\},\{d\},\{b, d\},\{a, b, d\},\{b, c, d\}\}$. Then $(S(X), E(X))$ is not semi-T 1 since $\{a, b, c\}, X \in S(X)$ such that $\{a, b, c\} \neq X$ and there does not exist a semi open set containing $\{a, b, c\}$ and not $x$.

In Willard's book [5], $\mathrm{T}_{0}$-identification spaces are discussed.

DEFINITION 3.2 Let $R$ be the equivalence relation on a space $(X, T)$ defined by $x$ Ry iff $\overline{\{x\}}=\overline{\{y\}}$. Then the $T_{0}$-identification space of $(X, T)$ is $\left(X_{0}, Q\left(X_{0}\right)\right.$ ), where $X_{0}$ is the set of equivalence classes of $R$ and $Q\left(X_{0}\right)$ is the decomposition topology on $x_{0}$, which is $T_{0}$. 
This author [9] used $\mathrm{T}_{0}$-identification spaces to show that hyperspaces of $R_{0}$ spaces, spaces which were first defined and investigated by Davis [10], are $T_{1}$. DEFINITION 3.3. A space $(X, T)$ is $R_{0}$ iff for each $0 \in T$ and $x \in 0$, $\overline{\{x\}} \subset 0$.

Since $T_{1}$ implies semi- $T_{1}$, then the hyperspace of each $R_{0}$ space is semi- $T_{1}$.

Semi open sets were used by Crossley and Hildebran [11] to define and investigate semi closed sets and semi closure.

DEFINITION 3.4. Let $(X, T)$ be a space and let $A, B \subset X$. Then $A$ is semi closed iff $X-A$ is semi open and the semi closure of $B$, denoted by scl $B$, is the intersection of all semi closed sets containing B.

This author [12] used semi closure to define and investigate semi- $\mathrm{T}_{0}-$ identification spaces.

DEFINITION 3.5. Let $R$ be the equivalence relation on a space $(X, T)$ defined

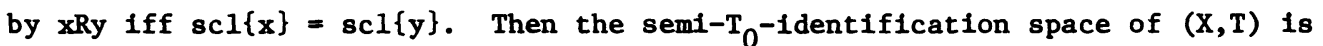
$\left(x_{S 0}, Q\left(X_{S 0}\right)\right.$, where $x_{S 0}$ is the set of equivalence classes of $R$ and $Q\left(X_{S 0}\right)$ is the decomposition topology on $\mathrm{x}_{\mathrm{S} 0}$, which is semi- $\mathrm{T}_{0}$.

This author [13] and [12] showed that the natural map $P:(X, T) \rightarrow\left(X_{0}, Q\left(X_{0}\right)\right)$ is continuous, closed, open, onto, and $P^{-1}(P(0))=0$ for all $0 \in T$ and that the natural map $P_{S}:(X, T) \rightarrow\left(X_{S 0}, Q\left(X_{S 0}\right)\right)$ is continuous, closed, open, onto, and $P_{S}^{-1}\left(P_{S}(0)\right)=0$ for all $0 \in S 0(X, T)$. These results are used to obtain the following result.

THEOREM 3.1. For a space $(X, T),(S(X), E(X)),\left(S\left(X_{0}\right), E\left(X_{0}\right)\right)$, and $\left(\mathrm{S}\left(\mathrm{X}_{\mathrm{SO}}\right), \mathrm{E}\left(\mathrm{X}_{\mathrm{SO}}\right)\right)$ are homeomorphic.

PROOF: Let $\mathrm{f}:(\mathrm{S}(\mathrm{X}), \mathrm{E}(\mathrm{X})) \rightarrow\left(\mathrm{S}\left(\mathrm{X}_{0}\right), \mathrm{E}\left(\mathrm{X}_{0}\right)\right)$ and let $\mathrm{f}_{\mathrm{S}}:(\mathrm{S}(\mathrm{X}), \mathrm{E}(\mathrm{X})) \rightarrow$ $\left(S\left(X_{S 0}\right), E\left(X_{S 0}\right)\right)$ defined by $f(F)=P(F)$ and $f_{S}(F)=P_{S}(F)$. Then $f$ and $f_{s}$ are homeomorphisms.

THEOREM 3.2. If $(X, T)$ is $R_{0}, G \in T$, and $F \in S(X)$ such that $F \cap \bar{G} \neq \emptyset$, then $S(\bar{G})=\overline{S(G)}$ and $F \in \overline{I(G)}$.

PROOF: Since $S(G) \subset S(\bar{G})$, which is closed, then $\overline{S(G)} \subset S(\bar{G})$. Let $A \in S(\bar{G})$. Let $\left\langle B_{i}\right\rangle_{i=1}^{P} \in B$ such that $A \in\left\langle B_{1}\right\rangle_{i=1}^{P}$. Then $A \subset \bar{G}$ and 
$\emptyset \neq A \Gamma_{i} B_{i} \subset \bar{G} \Gamma_{i} B_{i}$ for all $i \in N_{p}$, which implies $G \Gamma_{i} B_{i} \neq \emptyset$ for all $i \in N_{p}$. For each $i \in N_{p}$ let $x_{i} \in G \Gamma_{i} B_{i}$. Then $\overline{\left\{x_{i}\right\}} \subset G \Gamma_{i} B_{i}$ for all $i \in N_{p}$ and $\bigcup_{1} \in_{N_{P}} \overline{\left\{x_{1}\right\}} \in S(G) \cap\left\langle B_{1}\right\rangle_{1=1}^{P}$. Thus $A \in \overline{S(G)}$ and $S(\bar{G}) \subset \overline{S(G)}$, which Implies $S(\bar{G}) \stackrel{P}{=} \overline{S(G)}$.

Let $\left\langle U_{1}\right\rangle_{i=1}^{m} \in B$ such that $F \in\left\langle U_{i}\right\rangle_{i=1}^{m}$. Then FC $U_{1} \in_{m} U_{1} \in T$ and $F \cap \bar{G} \neq \emptyset$, which implies $G \cap\left({ }_{i \in N_{m}} U_{1}\right) \neq \emptyset$. Let $y \in G \cap\left({ }_{1 \in N_{m}} U_{1}\right)$ and for each $i \in N_{m}$ let $y_{i} \in B_{i}$. Then $\{\bar{y}\}^{m} \cup\left(U_{i \in N_{m}}\left\{\overline{y_{i}}\right\}\right) \in I(G) r_{1}<U_{i}>_{i=1}^{\frac{m}{m}}$. Hence, $F \in \overline{I(G)}$.

THEOREM 3.3. If $(X, T)$ is s-regular and $R_{0}$, then $(S(X), E(X))$ is semi-T 2 . PROOF: Let $A, B \in S(X)$ such that $A \neq B$. Then $A-B \neq \emptyset$ or $B-A \neq \emptyset$, say $B-A \neq \emptyset$. Let $x \in B-A$. Then there exists disjoint semi open sets 0 and $W$ such that $x \in 0$ and $A \subset W$. Let $U, V \in T$ such that $U \subset 0 \subset \bar{U}$ and $V \subset W \subset \bar{V}$. Then $I(U)$ and $S(V)$ are disjoint open sets, $B \in \overline{I(U)}$, and $A \in S(\bar{V})=\overline{S(V)}$, which implies $S(V) \cup\{A\}$ and $I(U) \cup\{B\}$ are disjoint semi open sets.

Maheshwari and Prasad [4] showed that every s-normal $R_{0}$ space is s-regular. This result can be combined with Theorem 3.3 to obtain the following corollary. COROLLARY 3.1. If $(X, T)$ is s-normal and $R_{O}$, then $(S(X), E(X))$ is semi- $T_{2}$. 


\section{REFERENCES}

1. LEVINE, N., Semi Open Sets and Semi Continuity in Topological Spaces, Amer. Math. Monthly, 70 (1963), 36-41.

2. MAHESHWARI, S. and PRASAD, R., Some New Separation Axioms, Ann. Soc. Sci. Bruxelles, 89 (1975), 395-402.

3. MAHESHWARI, S. and PRASAD, R., On s-Regular Spaces, Glasnik Mat. Ser. III, 10 (30) (1975), 347-350.

4. MAHESHWARI, S. and PRASAD, R., On s-Normal Spaces, Bull. Math. de la Soc. Sci. Math. de la R. S. de Roumanie, T 22(70) (1978), 27-30.

5. WILLARD, S., General Topology, Addison-Wesley Publishing Company, 1970.

6. CROSSLEY, S. and HILDEBRAND, S., Semi-Topological Properties, Fund. Math., $\underline{74}(1972), 233-254$.

7. FRINK, 0., Topology in Lattices, Trans. Amer. Math. Soc., 51 (1942), 569-582.

8. MICHAEL, E., Topologies on Spaces of Subsets, Trans Amer. Math. Soc., 71 (1951), 152-182.

9. DORSETT, C., $\mathrm{T}_{0}$-Identification Spaces and Hyperspaces, Ann. Soc. Sci. Bruxelles, 91 (1977), 200-206.

10. DAVIS, A., Indexed Systems of Neighborhoods for General Topological Spaces, Amer. Math. Month1y, 68 (1961), 886-893.

11. CROSSlEy, S. and HILDEBRAND, S., Semi-closure, Texas J. Science, 22 (1970), 99-112.

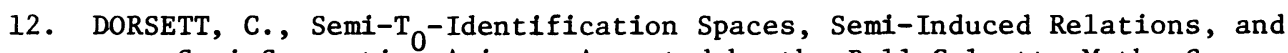
Semi Separation Axioms, Accepted by the Bull Calcutta Math. Soc.

13. DORSETT, C., $T_{0}$-Identification Spaces and $R_{1}$ Spaces, Kyungpook Math. J., $\underline{18(2)}(1998), 167-174$. 


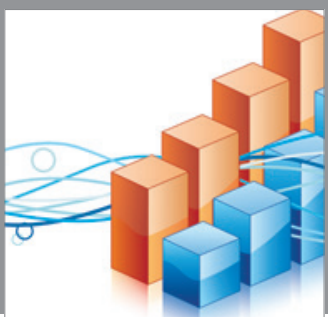

Advances in

Operations Research

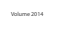

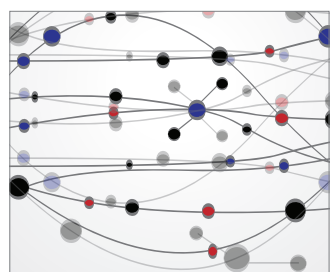

\section{The Scientific} World Journal
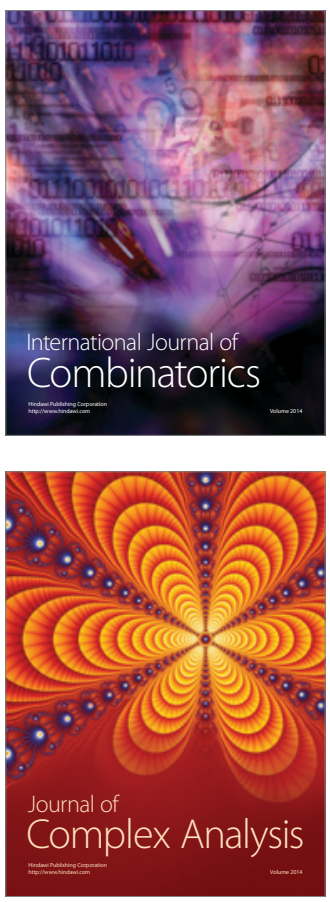

International Journal of

Mathematics and

Mathematical

Sciences
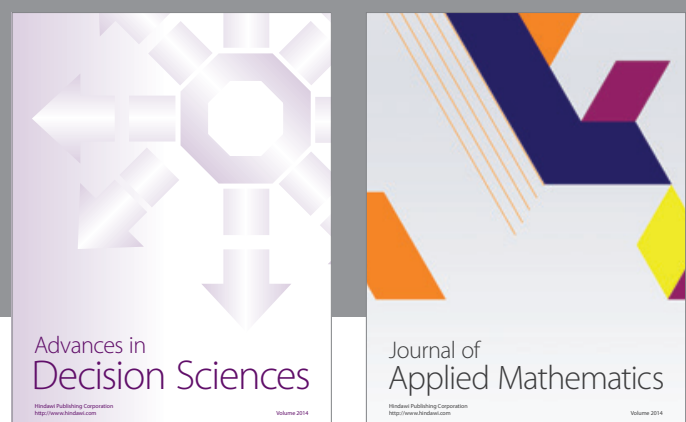

Journal of

Applied Mathematics
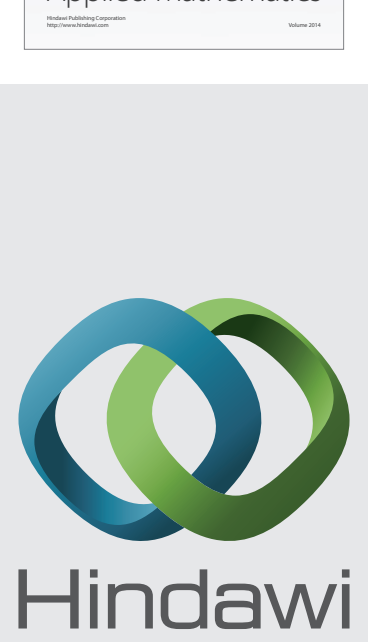

Submit your manuscripts at http://www.hindawi.com
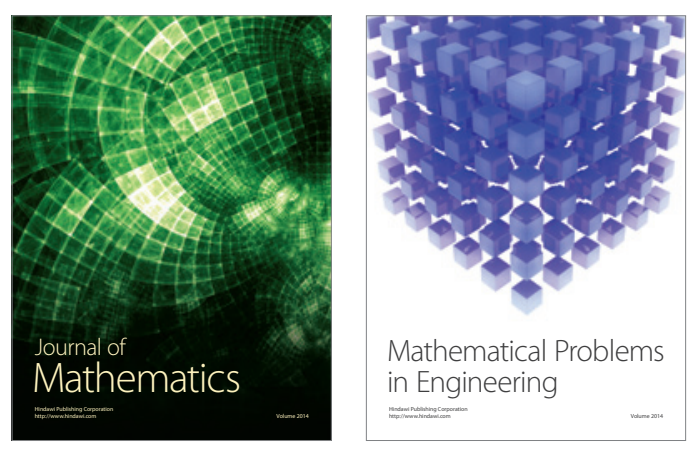

Mathematical Problems in Engineering
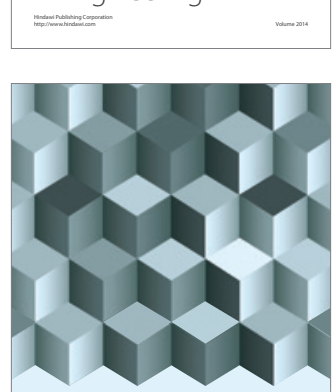

Journal of

Function Spaces
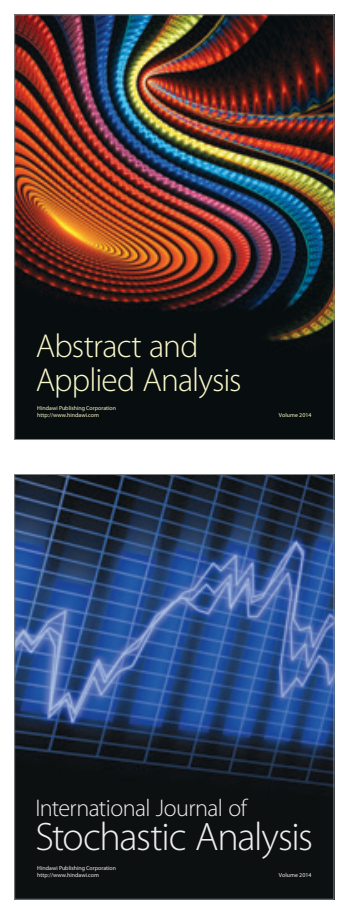

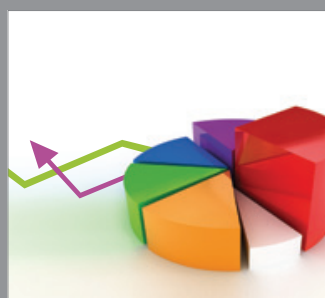

ournal of

Probability and Statistics

Promensencen
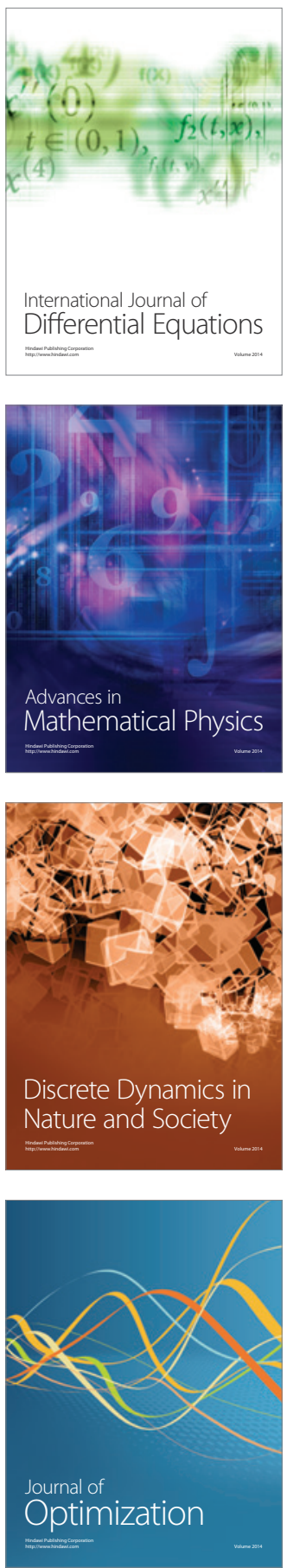